\title{
Factores asociados al rendimiento académico en estudiantes de medicina
}

\author{
Factors associated with academic performance in medical students
}

\author{
Alberto Vélez van Meerbeke y Claudia Natalí Roa González \\ Facultad de Medicina Universidad del Rosario, Bogotá, Colombia
}

Objetivos: Determinar y evaluar los factores que estuvieran incidiendo en el desempeño académico de nuestros estudiantes de primer semestre de medicina. Metodología: Se caracterizó la población en búsqueda de factores que se analizaron posteriormente para determinar asociación y predicción, a través de un modelo de regresión logística, del rendimiento académico final.

Resultados: Se analizaron 80 estudiantes de edades comprendidas entre 17 y 18, la mayoría mujeres, procedentes de Bogotá, de colegios mixtos, privados y monolingües. El grupo fue homogéneo por factores sociodemográficos, culturales, escolaridad y de motivaciones. Se detectaron rasgos de violencia intrafamiliar, de consumo de alcohol y cigarrillo pero no de drogas psicoactivas. Los resultados obtenidos en la prueba de aptitudes diferenciales y generales (BADyGs) del aprendizaje fueron bajos. El análisis mostró que el no leer como pasatiempo, la presencia de violencia intrafamiliar, el haber fumado marihuana, el provenir de un colegio mixto, el no haber realizado estudios profesionales, de quien se depende económicamente, las notas de biología, bioquímica y del promedio trimestral fueron los factores que se asocian con fracaso académico o pérdida de cupo. La variable que predice fracaso académico cuando se

Correspondencia:

Alberto Vélez van Meerbeke

Facultad de Medicina Universidad del Rosario

Calle 63D N²4-31

Bogotá, Colombia

Telefax: 57-1-3474570 ext 210

Correo Electrónico: avelez@urosario.edu.co controla por los otros factores incluidos en el modelo es el promedio trimestral y la que determina pérdida de cupo es la nota del laboratorio de bioquímica. Conclusiones: Aunque existen factores previos al ingreso que puedan explicar el desempeño académico, es importante evaluar el rendimiento durante el semestre para intervenir rápidamente y evitar el fracaso.

Palabras Clave: Educación Médica, Rendimiento académico, Fracaso académico, Éxito académico, CAGE, BADyGs.

Objectives: To determine and evaluate factors that affect academic performance in first semester medicine students

Methodology: The population was characterized in a search for factors that would later be studied in order to determine statistical association and prediction of final academic performance, using a logistic regression model.

Results: Eighty students, aged between 17 and 18, were analysed. The majority were women, from Bogotá, from mixed (i.e. not single-sex), private and monolingual schools. The group was homogeneous in terms of demographic, social and cultural factors, and of schooling and motivation. Features of interfamilial violence, alcohol and cigarette consumption (but not psychoactive drug use) were detected. The results obtained in the differential and general learning aptitude tests (BADyGs) were low. Factors associated with poor academic performance were: No interest in reading as a pastime; interfamilial violence; a history of smoking marijuana; mixed educational background; no history of premedical studies; economic dependence on one of the parents. Grades in biology and biochemistry and the quarterly average grades were the best 
predictors. The best predictor of poor academic performance when controlled by other factors included in the model was the quarterly average, and the best predictor of failing the academic semester was the biochemistry laboratory grade.

Conclusions: Although prior factors may explain academic performance before admission to Medical school, it is important to evaluate the performance during the first semester to identify cases in which rapid intervention is required in order to avoid academic failure.

Key words: Medical Education, academic performance, academic failure, academic success, $C A G E, B A D y G$.

\section{INTRODUCCIÓN}

El rendimiento académico ha sido definido como el cumplimiento de las metas, logros u objetivos establecidos en el programa o asignatura que está cursando un alumno ${ }^{1}$. Desde un punto de vista operativo, este indicador se ha limitado a la expresión de una nota cuantitativa o cualitativa y se encuentra que en muchos casos es insatisfactorio lo que se ve reflejado en la pérdida de materias, pérdida del cupo (mortalidad académica) o deserción.

Las estadísticas de la Facultad de Medicina de la Universidad del Rosario desde el segundo periodo académico de 1997 hasta el primer periodo de 2002 revelan que en promedio 105 estudiantes ingresan a la facultad semestralmente y $30 \%$ no superan el primer semestre por pérdida de materia, pérdida de cupo o deserción. Se hace necesario entonces, conocer el tipo de población para la cual va dirigida esta educación, evaluar el perfil del estudiante dentro del escenario político, sociodemográfico, económico y educativo en el cual se ha desarrollado y valorar sus necesidades, intereses y problemática para las desarrollar políticas necesarias para ayudar al mejor desempeño de los estudiantes.

Partiendo de esta premisa, se ha estudiado una serie de variables que puedan predecir el rendimiento universitario, entre ellos, los resultados previos en el colegio, las pruebas de estado, el examen de ingreso a la universidad, las aptitudes intelectuales, los factores psicosociales, los rasgos de personalidad y los factores emocionales, los hábitos de estudio y el interés vocacional ${ }^{1-5}$.

El presente estudio pretende evaluar la situación actual de los estudiantes que ingresan al primer semestre y los factores que influyen en su rendimiento académico. Esta información es necesaria para generar nuevas estrategias metodológicas para la adquisición de conocimiento científico y tecnológico, y estimular fundamentalmente el desarrollo de aptitudes, de valores, y particularmente la formación de un pensamiento crítico e independiente ${ }^{6}$.

\section{PARTICIPANTES, MATERIALES Y MÉTODOS}

El presente estudio se realizó en dos etapas: En la primera se caracterizó la población de estudiantes de primer semestre de la Facultad de Medicina, y en la segunda se realizó un estudio analítico para identificar la asociación entre el rendimiento académico y los factores analizados mediante un modelo de regresión logística.

\section{Participantes:}

Se incluyeron los estudiantes que ingresaron a primer semestre a la Facultad de Medicina en el segundo periodo de 2003 quienes de carácter voluntario y de manera anónima decidieron entrar en el estudio. Se excluyeron aquellos que estaban repitiendo una o más asignaturas de primer semestre en el periodo académico.

Para el cálculo del tamaño de muestra, se tomó la proporción de la asociación entre las calificaciones de secundaria y el rendimiento universitario durante la carrera, del trabajo de Humberto de Spinola ${ }^{1}$ en el cual $61 \%$ de los que tuvieron bajas notas presentaron mal rendimiento y $87 \%$ de los que tuvieron altas calificaciones tuvieron buen rendimiento.

El estudio fue analizado y aprobado por parte del Comité de Ética en Investigaciones de la Facultad de Medicina, debido a que trataba de un estudio con un grupo especial de sujetos, estudiantes de la Facultad de Medicina, en los que cabe suponer subordinación.

\section{Métodos}

Se midieron las siguientes variables independientes agrupadas en siete categorías: datos demográficos, datos de la admisión en la Universidad del Rosario, la composición familiar, los hábitos de estudio, los antecedentes escolares de secundaria, el examen de estado, la prueba neuropsicológica "Batería de aptitudes diferenciales y generales del aprendizaje" (superior) (BADyGs) ${ }^{7}$, y las notas parciales y finales de cada una de las asignaturas cursadas. Se indagó igualmente por el consumo de alcohol y de sustancias evaluando el riesgo de alcoholismo. Para 
esto se aplicó a todos los estudiantes que contestaron que consumía alcohol, el cuestionario CAGE (Cut por culpable de beber; Annoyed por molesto por la crítica; Guilty por culpable por beber y Eye-opener por necesidad de beber en la mañana) ${ }^{8-10}$. Como variables dependientes se tomaron el éxito o fracaso académico, definido este último como la pérdida de una o más materias, y la pérdida del cupo que se refirió a aquellos estudiantes que perdieron dos o más materias, que el promedio global fue menor de 3.3 o que se retiraron prematuramente de la Facultad.

Se realizó una prueba piloto con la presentación del proyecto a 14 estudiantes que cursaron primer semestre de Medicina en el primer periodo académico de 2003 para evaluar la encuesta.

\section{Instrumentos:}

Se utilizó la BADYG superior que es aplicable a población universitaria en los primeros años de carrera $^{7}$. Se escogieron las cuatro subpruebas más relacionadas con el objetivo del estudio basados adicionalmente en la ayuda de dos especialistas. Estas fueron "habilidad mental verbal" (HMV), "habilidad mental no verbal" (HMNV), comprensión verbal (CV) y razonamiento lógico (RL). Los puntajes se clasificaron en bajo, promedio bajo, promedio, promedio alto y alto en relación a lo encontrado por el autor?

Las asignaturas que los estudiantes cursan en el primer semestre de la Facultad son Biología Celular y Molecular (8 créditos académicos), Bioquímica Estructural (5 créditos), Promoción y Prevención (4 créditos) y Ética (3 Créditos). Las dos primeras asignaturas son de tipo teórico-práctico y la pérdida de ellas puede deberse a tener una nota final menor de 2.95 , una nota práctica menor de 2.95 o ambas. El promedio global para superar el semestre debe estar por encima de 3.3 .

\section{Análisis estadístico:}

En primera instancia, se realizó un análisis descriptivo de todas las variables según su escala de medición: proporciones de ocurrencia para variables cualitativas y medidas de tendencia central y de variabilidad para las continuas. Se establecieron diferencias entre grupos con respecto al sexo, edad y haber realizado previamente estudios profesionales (se refiere a cursos libres previos a la entrada en la Facultad, con asignaturas de la carrera). Se calculó la asociación de los factores de riesgo con las variables dependientes (éxito / fracaso y pérdida de cupo) utilizando razones de disparidad e intervalos de con- fianza del 95\% para evaluar la precisión de la estimación. Se analizó la correlación entre las variables numéricas con el coeficiente de correlación de Pearson. Finalmente se hizo un análisis de regresión múltiple bajo el método de paso a paso hacia delante para evaluar las variables que podían estar asociadas con la presentación de fracaso académico o pérdida de cupo. Su usó una probabilidad asociada menor del $10 \%$ como criterio de entrada al modelo.

\section{RESULTADOS}

\section{Descripción de la población:}

De los 120 alumnos que conformaron el primer semestre de la Facultad de Medicina en el primer periodo de 2003 se obtuvieron datos de 80 (66\%), los cuales cumplieron con los criterios de inclusión y exclusión y completaron las encuestas. El grupo en su composición correspondió al promedio de la Facultad con un predominio de mujeres, de edades comprendidas entre los 17 y 18 años, solteros y pertenecientes a estratos sociales altos. De igual manera, la composición y las relaciones familiares fue similar a la media nacional con 3 a 5 personas viviendo bajo el mismo techo $(82 \%)$ y se reconoce como jefe del hogar y se depende económicamente de uno o ambos progenitores en forma repartida. En general las relaciones intrafamiliares eran adecuadas pero se encontraron rasgos de violencia en 13 familias $(16 \%)$.

Los estudiantes realizaban muy pocas actividades durante su tiempo libre, de tipo cultural o deportivo pero si recreativas como ir al cine, reunirse con los amigos, leer y utilizar el Internet como pasatiempo. Por otro lado, sólo el $\mathbf{1 2 . 8 \%}$ de las personas trabajaba en su tiempo libre y de estos, dos lo hacían todos los días lo que estuvo relacionado con la pérdida de cupo.

El consumo de sustancias psicoactivas no fue un problema encontrado en los estudiantes pero si el alcohol, que se consumía con frecuencia por la gran mayoría (82.5\%), y hasta la embriaguez en 33 (50\%) sobretodo en los hombres $(\mathrm{p}<0.005)$. En ese sentido, 18 estudiantes estaban con sospecha de alcoholismo y seis podían ser catalogados como en riesgo de alcoholismo. De los estudiantes con cuestionario sospechoso o positivo para alcoholismo, 5 tuvieron mal rendimiento académico pero no se encontró asociación estadísticamente significativa.

Dentro de los parámetros necesarios para el ingreso a la Facultad (promedio ponderado del Bachillerato, examen de estado y entrevista), se 
encontró que la población era muy homogénea y no hubo ninguna asociación en lo que se refiere a pérdida de cupo o de asignatura.

81 personas contestaron la prueba de BADyGs. Los resultados encontrados estuvieron por debajo del promedio para la pueba, salvo en el razonamiento lógico. Sin embargo, y a pesar de estos resultados, no se obtuvo ninguna correlación entre los resultados y el rendimiento final, el éxito o fracaso académico ni la pérdida de cupo.

Se encontró que las calificaciones finales fueron superiores de forma significativa respecto a las trimestrales para el promedio general, biología, biología práctica, bioquímica y promoción y prevención . Del total de la población, 54 personas $(70 \%)$ pasaron el primer semestre sin pérdida de asignaturas con un promedio académico de 3,71 (+/- 0,23) . De éstos, sólo uno quedó con matrícula condicional por presentar un promedio académico entre 3,0 y 3,29. Dentro del grupo de fracaso académico fueron catalogadas 25 personas (32.5\%), 10 por haber perdido una asignatura (12.9\%), 13 el cupo (16.8\%) y 2 por retiro (2.5\%). Es de anotar que todas las personas que perdieron dos o más materias, perdieron el cupo.

\section{Análisis entre los factores estudiados}

y el rendimiento:

En el análisis bivariado se puede apreciar que aunque existen algunos factores previos al ingresos que se relacionan con mal rendimiento, las calificaciones durante la primera mitad del semestre son las que tienen una mayor asociación (tabla 1). Existen pocas diferencias cuando se realiza un análisis por sexo salvo con la variable violencia intrafamiliar en la que se observa una predominancia en el género masculino aunque esta asociación no necesariamente es conclusiva $(\mathrm{p}=0.038 \mathrm{OR}=0.19$; IC $95 \%=0.03-1.29$ ).

Lo mismo que con la anterior, el análisis de la pérdida de cupo muestra que la pérdida de algunas materias durante el semestre están más relacionadas con la pérdida de cupo que los factores previos (tabla 2). La dependencia económica y quien paga los estudios, aunque repartidos entre el padre, la madre o ambos están igualmente asociadas con este desenlace.

Bajo las características definidas en el plan de análisis para el estudio multivariado, se desprende que la variable más influyente en la predicción de la ocurrencia del fracaso académico cuando se controla por los otros factores incluidos es haber obtenido un promedio trimestral menor de 3.0. Las otras variables aunque importantes, no muestran una alta precisión en la estimación con los intervalos de confianza del $95 \%$ para los coeficientes de regresión (tablas 3,4 )

Del modelo en el que se toma pérdida de cupo como variable dependiente, se puede decir que sólo la pérdida de bioquímica-laboratorio da la posibilidad de una predicción de la pérdida de cupo cuando además se controla por la pérdida de biología tri-

Tabla 1: Factores asociados al fracaso académico

\begin{tabular}{l|l|l|l|l|l|l}
\hline Variable & Éxito n(\%) & Fracason(\%) & OR & IC 95\% & X2 & P \\
\hline No leer como pasatiempo & $13(24)$ & $12(48)$ & 3,1 & $1-9,3$ & 4,3 & 0,038 \\
Presencia de violencia intrafamiliar & $2(4)$ & $5(20)$ & 5,1 & $1,3-14,8$ & 7,3 & 0,007 \\
Haber fumado Marihuana & $2(4)$ & $4(16)$ & 6,8 & $1-56,4$ & 6,8 & 0,015 \\
Colegio mixto $*$ & $27(53)$ & $20(80)$ & 3,6 & $1-12,9$ & 5,2 & 0,022 \\
Biología trimestral $<3$ & $4(7.5)$ & $17(68)$ & 28 & $6,2-140,9$ & 31,8 & 0,000 \\
Biología practica trimestral $<3$ & $8(15)$ & $17(68)$ & 12,9 & $3,5-49,9$ & 22,0 & 0,000 \\
Bioquímica trimestral <3 $(1)$ & $15(28)$ & $23(95)$ & 58,3 & $7,1-1263$ & 30,1 & 0,000 \\
Bioquímica laboratorio $<3$ & $2(4)$ & $13(54)$ & 27,8 & $4,8-212$ & 25 & 0,000 \\
Promedio trimestral<3 & $1(2)$ & $17(71)$ & 55,7 & $9-453$ & 37,4 & 0,000 \\
No realizar estudios profesionales $* *$ & $35(66)$ & $22(88)$ & 3,8 & $0,9-18,5$ & 4,5 & 0,038 \\
\hline
\end{tabular}

OR: Razón de disparidad; IC: intervalo de confianza

* Colegio mixto se refiere a un colegio en el que acuden niños de ambos sexos.

** Esta variable se incluye aunque el intervalo de confianza no es significativo. 


\section{Tabla 2: Factores de riesgo asociados con pérdida de cupo}

\begin{tabular}{l|l|l|l|l|l|l|}
\hline Variable & No pérdida $\mathrm{n}(\%)$ & Pérdida $\mathrm{n}(\%)$ & OR & IC 95\% & Chi2 & P \\
\hline Dependencia económica (1) & & & & & 6,4 & 0,041 \\
Quien paga los estudios (1) & & & & & 8,3 & 0,039 \\
Necesidad de trabajar & $6(9)$ & $4(36)$ & 5,52 & $1-31,11$ & 5,9 & 0,015 \\
Colegio mixto & $36(55)$ & $10(91)$ & 8,8 & $1,05-193,29$ & 5,5 & 0,018 \\
Haber fumado Marihuana & $4(6)$ & $4(36)$ & 9,67 & $1,44-7,32$ & 9,3 & 0,002 \\
Biología trimestral <3 & $11(17)$ & $9(82)$ & 22,09 & $3,62-173,99$ & 20,4 & 0,000 \\
Biología practica trimestral <3 & $15(23)$ & $9(82)$ & 15 & $2,53-113,95$ & 15 & 0,000 \\
Bioquímica trimestral <3 & $27(42)$ & $10(91)$ & 14,07 & $1,66-311,37$ & 9,2 & 0,002 \\
Bioquímica laboratorio <3 & $6(9)$ & $8(73)$ & 26,22 & $4,51-177,23$ & 25,2 & 0,000 \\
Promedio trimestral<3 & $7(11)$ & $10(91)$ & 96,57 & $9,43-2414,5$ & 37,2 & 0,000 \\
\hline
\end{tabular}

Nota (1): No se presentan los porcentajes ni se calcula la razón de disparidad debido al número de categorías que tiene la variable.

\section{Tabla 3: Factores que explican el fracaso académico}

\begin{tabular}{l|l|l|l|l|}
\hline Fracaso académico & Coeficiente & Error estándar & $\mathrm{P}$ & Intervalo de confianza 95\% \\
\hline Promedio trimestral $<3$ & 4,433924 & 1,542838 & 0,004 & 1,$410 ; 7,458$ \\
Biología trimestral $<3$ & 4,218878 & 1,732186 & 0,015 & 0,$823 ; 7,614$ \\
Violencia intrafamiliar & $-4,921582$ & 1,890114 & 0,009 & $-8,626 ;-1,217$ \\
Leer como pasatiempo & 3,783106 & 1,647628 & 0,022, & 0,$554 ; 7,012$ \\
$\mathrm{~N}^{\circ}$ horas utilizadas para estudiar & $-0,3886991$ & 0,157771 & 0,010 & $-0,686 ;-0,091$ \\
Haber fumado Marihuana & $-4,918772$ & 2,742112 & 0,073 & $-10,293 ; 0,456$ \\
Cons & $-3,479763$ & 1,694963 & 0,040 & $-6,802 ;-0,158$ \\
\hline
\end{tabular}

\section{Tabla 4: Peso de las variables según modelo}

\begin{tabular}{l|l|l|l|l|}
\hline Modelo & $\mathrm{N}^{\circ}$ observaciones & R2 ajustado (\%) & Log likelihood & $\mathrm{P}$ \\
\hline $\begin{array}{l}\text { Promedio trimestral }<3 \\
\text { Biología trimestral }<3 \\
\text { Violencia intrafamiliar } \\
\begin{array}{l}\text { No leer como pasatiempo } \\
\mathrm{N}^{\circ} \text { horas utilizadas para estudiar } \\
\text { Haber fumado Marihuana }\end{array}\end{array}$ & 72 & 73,3 & $-11,827$ & 0,000 \\
& & & & \\
\hline
\end{tabular}


Tabla 5: Factores que explican la pérdida de cupo

\begin{tabular}{l|l|l|l|l|}
\hline Pérdida de cupo & Coeficiente & Error estándar & $\mathrm{P}$ & Intervalo de confianza 95\% \\
\hline Bioquímica laboratorio $<3$ & 3,440135 & 1,13978 & 0,003 & 1,$206 ; 5,674$ \\
Biología trimestral $<3$ & 2,291403 & 0,9814965 & 0,020 & 0,$368 ; 4,215$ \\
Con cuantas personas vive & 0,9510115 & 0,5015451 & 0,058 & $-0,032 ; 1,934$ \\
Cons & $-4,987271$ & 2,140532 & 0,020 & $-9,183 ;-, 791$ \\
\hline
\end{tabular}

Tabla 6: Peso de las variables que explican pérdida de cupo según modelo

\begin{tabular}{l|l|l|l|l|}
\hline Modelo & $N^{\circ}$ observaciones & R ajustado (\%) & Log likelihood & P \\
\hline $\begin{array}{l}\text { Bioquímica laboratorio }<3 \\
\text { Biología trimestral }<3 \\
\text { Con cuantas personas vive }\end{array}$ & 75 & 53,41 & $-15,364$ & 0,000 \\
\hline
\end{tabular}

mestral y el numero de personas con quienes vive el estudiante (tablas 5, 6).

Dada la importancia de las notas hasta trimestrales encontradas en el modelo final y la poca presencia de factores previos que explicaran la pérdida de cupo o el fracaso académico, se decidió realizar un modelo tomando como variable dependiente la pérdida del promedio trimestral. La media del promedio trimestral fue de 3,3 $(+/$ _ 0,48) con un rango que osciló entre 1,24 y 4,29 y presentaron fracaso 19 estudiantes. Los factores que tuvieron asociación con esta variable fueron el haber estado en colegio mixto $(p=0,006)$; la presión para estudiar medicina $(\mathrm{p}=0,042)$; el haber fumado marihuana $(\mathrm{p}=0,009)$ y el riesgo de ser alcohólico según la encuesta CAGE ( $p=0,033)$. En el análisis multivariado, se evidenció que el modelo es explicado básicamente por el tipo de colegio en el que estudió el alumno (mixto) y el haber fumado marihuana pero el ajuste fue muy bajo ( $\mathrm{R}$ ajustado $=20 \%$ ) lo cual podría significar que la explicación es muy débil y por este motivo no se encuentra ninguna de esta variables relacionadas con el fracaso y la pérdida de cupo. Esto se evidencia en la realización de una prueba de correlación de Spearman con la falta de correlación entre el promedio de notas trimestrales y el éxito o fracaso final $(\mathrm{p}=0.0000)$.

\section{DISCUSIÓN}

La población de estudiantes de primer semestre de Medicina ingresa a la Facultad por medio de un proceso que involucra parámetros homogéneos de selección, lo que hace que el grupo estudiantil tenga unas características similares desde el punto de vista académico con promedios de notas de bachillerato y exámenes de estado por encima del promedio nacional. La Universidad por su lado es de carácter privado y con las implicaciones asociadas a esto, hace que la población que ingresa tenga igualmente unas características socioeconómicas homogéneas. Esto influye en la realización de un análisis comparativo que busca diferencias que pudieran explicar el rendimiento académico en los primeros semestres de carrera.

Por lo tanto, con relación a estos factores demográficos no hubo ninguna diferencia estadísticamente significativa con el rendimiento académico, en contraste con otros estudios en los que se estipula que las condiciones socioeconómicas, el género, la procedencia, el continuar en la casa paterna y la educación de los padres influyen de manera importante ${ }^{11-13}$. Cabe resaltar que al ser pocos estudiantes los que tienen estrato socioeconómico bajo no fue posible establecer si este factor incide en el rendimiento académico como lo sugiere Mizala en su estudio ${ }^{11}$. De forma similar, se encuentra una gran influencia de las actitudes, conductas y escolaridad de los padres y en términos generales la familia, sobre el aprendizaje de los niños y jóvenes en desarrollo ${ }^{13}$ al contrario de lo encontrado en este estudio, por la misma razón expuesta con anterioridad de similitud de la muestra. Sí se encontraron algunas diferencias, en lo referente a pérdida de cupo 
en aquellos casos en los que la dependencia económica y el pago de los estudios eran dados sólo por uno de los progenitores. Es de gran importancia resaltar que dentro de este aspecto, el fracaso académico esta relacionado con rasgos de violencia intrafamiliar los cuales se encontraron en el $16 \%$ de la población. Es claro que estar en un hogar en el que el trato entre la pareja es deficiente, puede influir negativamente en el medio ambiente en que se desarrolla el estudiante e perturbar su aprendizaje ${ }^{14}$.

El porcentaje de estudiantes que trabaja en su tiempo libre no es importante pero vale la pena resaltar que es una actividad que requiere de una gran carga horaria lo que esta influyendo de forma significativa con muy bajo rendimiento académico posiblemente por incumplimiento en las tareas, asistencia irregular, desconcentración en las clases y apatía a las actividades del aula por cansancio ${ }^{15}$.

Uno de los principales problemas de salud pública en la población joven es el consumo de drogas o cualquier tipo de sustancias psicoactivas llegando a cifras alarmantes que afectan alrededor del $50 \%$ de esta población ${ }^{16-17}$. Estas cifras contrastan con los hallazgos de este estudio en el que no se detectó consumo de cocaína, o pepas de uso no médico, y el consumo de marihuana está presente en el $10 \%$ de la población aunque la mayoría niega haber consumido dentro del último año. Una de las posibles explicaciones de estos resultados puede ser que el inicio del consumo de estas sustancias sea progresivo a medida que se avanza en la carrera o en edad y por otro lado pudo haber existido algún grado de temor al contestar este tipo de preguntas ${ }^{17-18}$.

Por otro lado, el consumo de alcohol presentó una alta incidencia en la población estudiada sobretodo en hombres. Más del $80 \%$ habían bebido dentro del último año y de estos un porcentaje importante lo habían hecho en el último mes o semana llegando a la embriaguez parte de ellos. Con la encuesta de CAGE se evidenció que $26 \%$ tenía sospecha y $8 \%$ estabá en alto riesgo de alcoholismo ${ }^{8}$. En un estudio de Secretaría de Programación para la Prevención de la Drogadicción y la Lucha contra el Narcotráfico en Argentina, se encontró que aquellos alumnos que presentaron un porcentaje mayor de consumo actual de alcohol son quienes manifestaron haber tenido una o varias veces problemas de aprendizaje y repitieron algún grado o curso o tuvieron problemas de disciplina ${ }^{18}$. En este sentido, uno de los propósitos de este estudio es realizar una intervención con estos alumnos, evitar que el consumo aumente y vaya en detrimento de su rendimiento académico.
El factor de satisfacción personal juega un papel importante en desempeño académico de los estudiantes $^{19}$. En este estudio se evidencia que la gran mayoría de los estudiantes se encuentra satisfechos con la elección que hicieron en cuanto a carrera y universidad sin presión por parte de los padres aunque aproximadamente el $30 \%$ de la población encontró dificultad en la adaptación al cambio colegio universidad.

La prueba de BADyGs ofrece un perfil de rendimiento aptitudinal que se compagina con la estructura de la inteligencia y en donde las sub-pruebas aplicadas permiten evaluar la habilidad para clasificar, deducir, analizar e integrar conceptos verbales y numéricos, síntesis deductiva, conocimiento de vocabulario y facilidad en el reconocimiento de relaciones verbales analógica. Llama la atención los bajos resultados obtenidos por los estudiantes del estudio en cada una de las subpruebas, predominando calificaciones bajas o promedio-bajas en mas del $50 \%$ de los alumnos. Ninguna de las personas que contestó la prueba logró terminar completamente las cuatro subpruebas a pesar que el tiempo suministrado para cada prueba es suficiente para que acaben aproximadamente el $20 \%$ de los sujetos ${ }^{7}$. Esto puede ser reflejo de una falta de comprensión por parte de los estudiantes, de que su bajo nivel no les permitió hacer estas pruebas colectivas o de que no quisieron hacerla correctamente debido a una posible falta de interés por los resultados. A pesar de lo anterior, vemos que aunque no se evidenció una relación entre los hallazgos y el rendimiento académico, existe una deficiente habilidad en las aptitudes evaluadas. No obstante, los resultados en la prueba de BADyGs muestran que los estudiantes egresan de su educación secundaria con bases poco sólidas en habilidades intelectuales lo que podría afectar el rendimiento académico en asignaturas que requieran habilidades de mayor complejidad como son interpretar, explicar, analizar, deducir y comparar.

Se evidenció que las notas promedio obtenidas en cada una de las materias están en un rango entre 3.05 y 3.77 para trimestrales con un aumento significativo en los promedios para finales, con notas en un rango entre 3.16 y 3.90. Esta diferencia de resultados obtenidos antes y después de trimestrales puede estar relacionada con la adaptación de los alumnos al método y régimen de estudio universitario o a una mayor preocupación por los resultados académicos obtenidos.

$\mathrm{Al}$ hacer el análisis de asociación entre los factores estudiados y el rendimiento académico se evidenció que de los factores que muestran relación estadísti- 
camente significativa con fracaso académico están: presencia de violencia intrafamiliar, consumo de marihuana, proceder de un colegio que recibe estudiantes de ambos sexos y tener calificaciones en trimestrales inferior a 3.0 en biología, bioquímica y en promedio. Al analizar en particular la "pérdida de cupo" se evidenció que la necesidad de trabajar, proceder de colegio mixto, haber fumado marihuana y las notas en trimestrales en biología y bioquímica influyen de manera estadísticamente significativa.

Por otro lado, variables como leer por pasatiempo $\mathrm{y}$ haber realizado estudios profesionales influyen positivamente con el éxito académico lo que puede ser consecuencia de que en esa etapa el estudiante recibe asignaturas cuyos contenidos son más afines con lo que necesitan para cursar las asignaturas de primer semestre lo que puede generar herramientas útiles en el aprendizaje de las mismas ${ }^{19}$.

En la literatura esta descrito que los hombres tienen un rendimiento más alto que las mujeres, también el rendimiento es superior si el alumno vive con sus padres ${ }^{11-13}$. Sin embargo en este estudio no se encontró una correlación entre estos factores y el rendimiento académico.

En cuanto a la predicción del rendimiento académico tanto a la mitad del semestre como al final, son pocos de los factores analizados en este estudio los que logran explicarlo. Teniendo en cuenta que muchas de las variables analizadas son factores previos al ingreso y que como se explicó anteriormente el proceso de selección de admisión a la Facultad hace que esta población sea homogénea, las características que logran explicar la diferencia en el rendimiento académico se relacionan con el desempeño de los estudiantes en el transcurso del semestre (primer trimestre académico). El modelo final de predicción revela una asociación para fracaso académico con el promedio obtenido en trimestrales (estos datos son independientes de los obtenidos al final según la prueba de Spearman) y para pérdida de cupo con la nota de bioquímica práctica cuando se controlan con otras variables. Es importante tener en cuenta que el número de observaciones en el modelo, no permitió dividir la tipo en dos para realizar la validación del mismo.

En conclusión, vemos que las causas determinantes del fracaso escolar no quedan plenamente establecidas. Sería conveniente realizar investigaciones complementarias a fin de determinar otras posibles causas no analizadas en este estudio que pueden estar generando deficiencias en el rendimiento académico de determinados estudiantes, lo que permitirá contar con mayores elementos a la hora de brindar ayuda a los alumnos que así lo necesiten. Sería importante analizar si el rendimiento académico esta influenciado. por factores tales como el tipo de profesor, la metodología utilizada para la enseñanza, los índices de capacidad de atención de los estudiantes, entre otros como lo demuestran estudios realizados en otros países al inicio de primer semestre de la carrera ${ }^{20}$.

\section{AGRADECIMIENTOS}

A las Dras Maria Victoria Montes y Claudia Talero por su asesoría en las pruebas psicolingüisticas; a la Dra. Maria Nelsy Rodriguez por la asesoría estadística. A los estudiantes de primer semestre por la colaboración en realizar las encuestas y pruebas.

\section{BIBLIOGRAFÍA}

1. Humberto de Spinola BR. Rendimiento académico y factores psicosociales en los ingresantes a la carrera de medicina - UNNE. Revista Paraguaya de Sociología 1990;78:143-167.

2. Álvarez MT, García H. Factores que predicen el rendimiento universitario. primera edición ed. San Juan de Pasto: Universidad de Nariño; 1996.

3. Musayón FY. Relación entre el Puntaje de Ingreso y el Rendimiento Académico en el Segundo año de las alumnas de Enfermería ingresantes entre los años 1,994 - 1,997 en una Universidad Peruana. Revista Universidades 2001;22.

4. Cascón I. Predictores del rendimiento académico en alumnos de primero y segundo de BUP. In: Disponible en URL:

http://www3.usal.es/ inico/investigacion/jornadas/ jornada2/comun/c19.html]. 2000; 2000.

5. Fergusson E, James D, Madeley L. Factors associated with success in medical school: systematic review of the literature. BMJ 2002;324:952-957.

6. OMS. Informe Conferencia Internacional sobre atención primaria de la Salud (Alma Ata). In; 1978; 1978.

7. Yuste Hernanz C. BADYG S: Manual Técnico. Madrid: IMPRESA; 1983.

8. Ewing JA. Detecting alcoholism: The CAGE questionnaire. JAMA 1984;252(1905-1907).

9. Mayfield DG, McLeod G, Hall P T. The CAGE questionnaire: Validation of a new alcoholism screening instrument. Am J Psychiatry 1974;131:1121-1123.

10. Rodríguez-Martos A, Navarro RM, Vecino C, Pérez R. Validación de los cuestionarios KFA (CBA) y CAGE para el diagnóstico del alcoholismo. Drogalcohol 1986; 11:132-139. 
11. Mizala A, Romaguera P, Reinaga T. Factores que inciden en rendimiento escolar en Bolivia. Disponible en URL:

http://www.itam.mx/lames/papers/contrses/romaguer.

12. Rosete Mohedano MG. Salud mental vs. rendimiento académico en alumnos de las carreras de: medicina, psicología y odontología de la FES Zaragoza. In: Seminarios de diagnósticos locales; 2003; Zaragoza: Facultad de Estudios Superiores Zaragoza; 2003.

13. Andrade M, Miranda C, Freixas I. Rendimiento académico y variables modificables en alumnos de 2 do medio de liceos municipales de la Comuna de Santiago. Revista de Psicología Educativa, 2000; 6(N. $\left.{ }^{\circ} 2\right)$ :ver en URL: http://www.unesco.cl/pdf/publicac/rendimiento.pdf.

14. Braverman R. Tipología Familiar Relacionada con Aspectos Psico-sociales-pedagógicos. Mèxico: UNAM; 1986.

15. González E. Jóvenes trabajadores, con bajo rendimiento escolar, en La hora Nacional. 5 de noviembre de 2002. La Hora Disponible en URL:

http://www.lahora.com.gt/02/11/05/paginas/nac_1. htm\#n1 2002.
16. Ministerio de Salud de Colombia. Estudio Nacional de Salud Mental y consumo de sustancias psicoactivas Colombia 1993. $2^{\circ}$ ed. Bogotá: Oficina de Comunicaciones Ministerio de Salud; 1995.

17. Allison K, Adlaf E, Lalomiteanu A. Predictors of health risk behaviours among young adults: Analysis of the National Population Health Survey. Can J Public Health 1999;90(2):85-89.

18. Ahumada G. Consumo de alcohol en jóvenes: Secretaría de Programación para la Prevención de la Drogadicción y la Lucha contra el Narcotráfico (SEDRONAR); 2003. Report No.: Disponible en URL: http://www.sedronar.gov.ar/s_institucional/links/ Encuestas_nacionales/nuevo1.htm.

19. Carrión Pérez E. Validación de características al ingreso como predictores del rendimiento académico en la carrera de medicina. Rev Cubana Educ Med Sup 2002; 16 (1):5-18.

20. Hidalgo de Paz A, Gil León M, Rodríguez Rodríguez E. Capacidad de atención y resultados docentes de estudiantes de primer año de medicina. Rev Cubana Educ Med Sup 2001;15(3):273-8. 